\title{
Parameter optimization for constructing competing endogenous RNA regulatory network in glioblastoma multiforme and other cancers
}

\author{
Yu-Chiao Chiu ${ }^{1,2 \dagger}$, Tzu-Hung Hsiao ${ }^{2,3 \dagger}$, Yidong Chen ${ }^{2,4^{*}}$, Eric Y Chuang ${ }^{1,5^{*}}$
}

From IEEE International Workshop on Genomics Signal Processing and Statistics (GENSIPS) 2013

Houston,TX, USA. 17-19 November 2013

\begin{abstract}
Background: In addition to direct targeting and repressing mRNAs, recent studies reported that microRNAs (miRNAs) can bridge up an alternative layer of post-transcriptional gene regulatory networks. The competing endogenous RNA (ceRNA) regulation depicts the scenario where pairs of genes (ceRNAs) sharing, fully or partially, common binding miRNAs (miRNA program) can establish coexpression through competition for a limited pool of the miRNA program. While the dynamics of ceRNA regulation among cellular conditions have been verified based on in silico and in vitro experiments, comprehensive investigation into the strength of ceRNA regulation in human datasets remains largely unexplored. Furthermore, pan-cancer analysis of ceRNA regulation, to our knowledge, has not been systematically investigated.
\end{abstract}

Results: In the present study we explored optimal conditions for ceRNA regulation, investigated functions governed by ceRNA regulation, and evaluated pan-cancer effects. We started by investigating how essential factors, such as the size of miRNA programs, the number of miRNA program binding sites, and expression levels of miRNA programs and ceRNAs affect the ceRNA regulation capacity in tumors derived from glioblastoma multiforme patients captured by The Cancer Genome Atlas (TCGA). We demonstrated that increased numbers of common targeting miRNAs as well as the abundance of binding sites enhance ceRNA regulation and strengthen coexpression of ceRNA pairs. Also, our investigation revealed that the strength of ceRNA regulation is dependent on expression levels of both miRNA programs and ceRNAs. Through functional annotation analysis, our results indicated that ceRNA regulation is highly associated with essential cellular functions and diseases including cancer. Furthermore, the highly intertwined ceRNA regulatory relationship enables constitutive and effective intra-function regulation of genes in diverse types of cancer.

Conclusions: Using gene and microRNA expression datasets from TCGA, we successfully quantified the optimal conditions for ceRNA regulation, which hinge on four essential parameters of ceRNAs. Our analysis suggests optimized ceRNA regulation is related to disease pathways and essential cellular functions. Furthermore, although the strength of ceRNA regulation is dynamic among cancers, its governing functions are stably maintained. The findings of this report contribute to better understanding of ceRNA dynamics and its crucial roles in cancers.

\footnotetext{
* Correspondence: cheny8@uthscsa.edu; chuangey@ntu.edu.tw

+ Contributed equally

${ }^{1}$ Graduate Institute of Biomedical Electronics and Bioinformatics, National

Taiwan University, Taipei, Taiwan

${ }^{2}$ Greehey Children's Cancer Research Institute, University of Texas Health

Science Center at San Antonio, San Antonio, Texas, USA

Full list of author information is available at the end of the article
}

(c) 2015 Chiu et al; licensee BioMed Central Ltd. This is an Open Access article distributed under the terms of the Creative Commons Attribution License (http://creativecommons.org/licenses/by/4.0), which permits unrestricted use, distribution, and reproduction in any medium, provided the original work is properly cited. The Creative Commons Public Domain Dedication waiver (http:// creativecommons.org/publicdomain/zero/1.0/) applies to the data made available in this article, unless otherwise stated. 


\section{Background}

A group of short single-stranded RNAs, namely microRNAs (miRNAs), has been widely investigated in this decade. With an average length of 22 nucleotides only, miRNAs are not protein coding transcripts. Instead, they fulfill the role of regulators of gene expression by complementarily binding to 3 ' untranslated regions ( 3 ' UTRs) of target mRNA transcripts [1,2]. According to existing biological evidence, the binding of miRNAs on mRNA can cause mRNA degradation or suppression of translation, and may affect expression of up to one third of the protein coding genes in humans [2]. In cancers, the dysregulation of miRNAs has been proven to be involved in oncogenesis (reviewed in [3]), tumor progression $[4,5]$, and clinical outcomes, such as patient survival $[6,7]$. With advances in next-generation sequencing, a great number of novel miRNAs have been identified and deposited in the public database miRBase [8], increasing the complexity of miRNA regulation.

Recently, reports postulated and experimentally validated that miRNAs can serve as an alternative layer of post-transcriptional gene-gene regulation, namely the competing endogenous RNAs (ceRNAs) [9-11]. Pairs of genes (ceRNAs) fully or partially sharing common binding miRNAs can establish crosstalk with each other through competition for a limited pool of the common miRNAs (miRNA programs; abbreviated as miRP). When expression level of one ceRNA rises (or decreases) in cells, it attracts (or releases) the targeting miRNAs away from (or toward) the other ceRNAs, and in turn has protective (or degradative) effects on expression of the other ceRNA partners. In other words, this postulation provides the scenario that genes can, facilitated by miRNAs, regulate each other without direct interaction. Through bioinformatic analysis and in vitro experiments on the tumor suppressor gene PTEN, previous studies suggested that ceRNAs of PTEN, e.g., VAPA and ZEB2, can possess tumor-suppressive properties by modulating (i.e. coexpressing with) PTEN expression levels in a miRNA-dependent while protein-coding independent manner [9-11]. Through in silico analysis of glioblastoma gene expression datasets, our recent study further demonstrated that ceRNA regulation, while only accounting for a small portion of global gene regulation, plays an essential role in transient cellular responses to dynamic inter-cellular signals [12]. Taken together, these observations have revealed that ceRNA regulation provides an alternative mechanism of gene regulation in essential cellular processes and functions. To address the optimal cellular conditions for ceRNA regulation, several recent studies used the mathematical massaction simulation [13,14] and cell line experiments [13] to demonstrate the dependency of ceRNA regulation on the dosages (i.e., cellular concentration) of both ceRNAs and miRPs, and number of miRNA response elements, suggesting the dynamic and condition-specific properties of ceRNA regulation in vitro.

Realizing that biological processes typically involve more complex mechanisms in vivo than in vitro, in the first part of this study we investigate the optimal conditions of ceRNA regulation in expression datasets derived from clinical samples. The optimal conditions may depend on the following essential factors: 1) Size of miRNA programs, 2) Number of miRNA program binding sites, 3) Expression level of miRNA programs, and 4) Expression level of ceRNAs. Here we developed an analytic scheme for determining whether these factors affect strength of ceRNA regulation. By integrating four factors' effects, the biological functions governed by optimal ceRNA regulation can be elucidated. On the other hand, while pan-cancer genomic analysis has been widely utilized to reveal tumor-specific and distinct molecular signature to better understand cancer heterogeneity $[15,16]$, pan-cancer analysis of ceRNA regulation, to our knowledge, has not been systematically explored. Collectively, the present study provides a systematic investigation of optimal conditions for ceRNA regulation, explores associated biological functions, and conducts pan-cancer analysis of ceRNAs in four cancer types.

\section{Results}

\section{Model overview and data preparation}

The proposed study aims to comprehensively explore optimal conditions for ceRNA regulation, to investigate functions governed by ceRNA regulation, and to evaluate pan-cancer effects. We started by investigating how essential factors, such as the size of miRNA programs, the number of miRNA program binding sites, and expression levels of miRNA programs and ceRNAs affect the ceRNA regulation in tumor samples from glioblastoma multiforme (GBM) patients. Here we chose GBM as the model cancer type because it is one of the most frequently studied cancers in investigating ceRNA regulation $[10,11]$. The analysis flowchart is illustrated in Figure 1. We analyzed 481 tumor sets with tumor-matched mRNA and miRNA expression profiles from TCGA [17]. Based on the definition of previous studies, we defined putative ceRNA pairs as two genes sharing any number of common predicted targeting miRNAs. Recruiting the prediction data from the TargetScan algorithm [2,18], we identified 47,451,423 putative ceRNA pairs with least one common targeting miRNA, comprising 10,872 ceRNAs (genes). For each of the putative ceRNA pairs, the pairwise correlation coefficient of gene expression profiles was computed (Figure 1A). Varying size of miRNA program, for example, generated multiple cumulative distribution functions (CDFs) of correlation coefficients. We then performed the goodness-of-fit tests (Kolmogorov-Smirnov test; K-S test) among the CDFs to pinpoint whether or not the optimal 


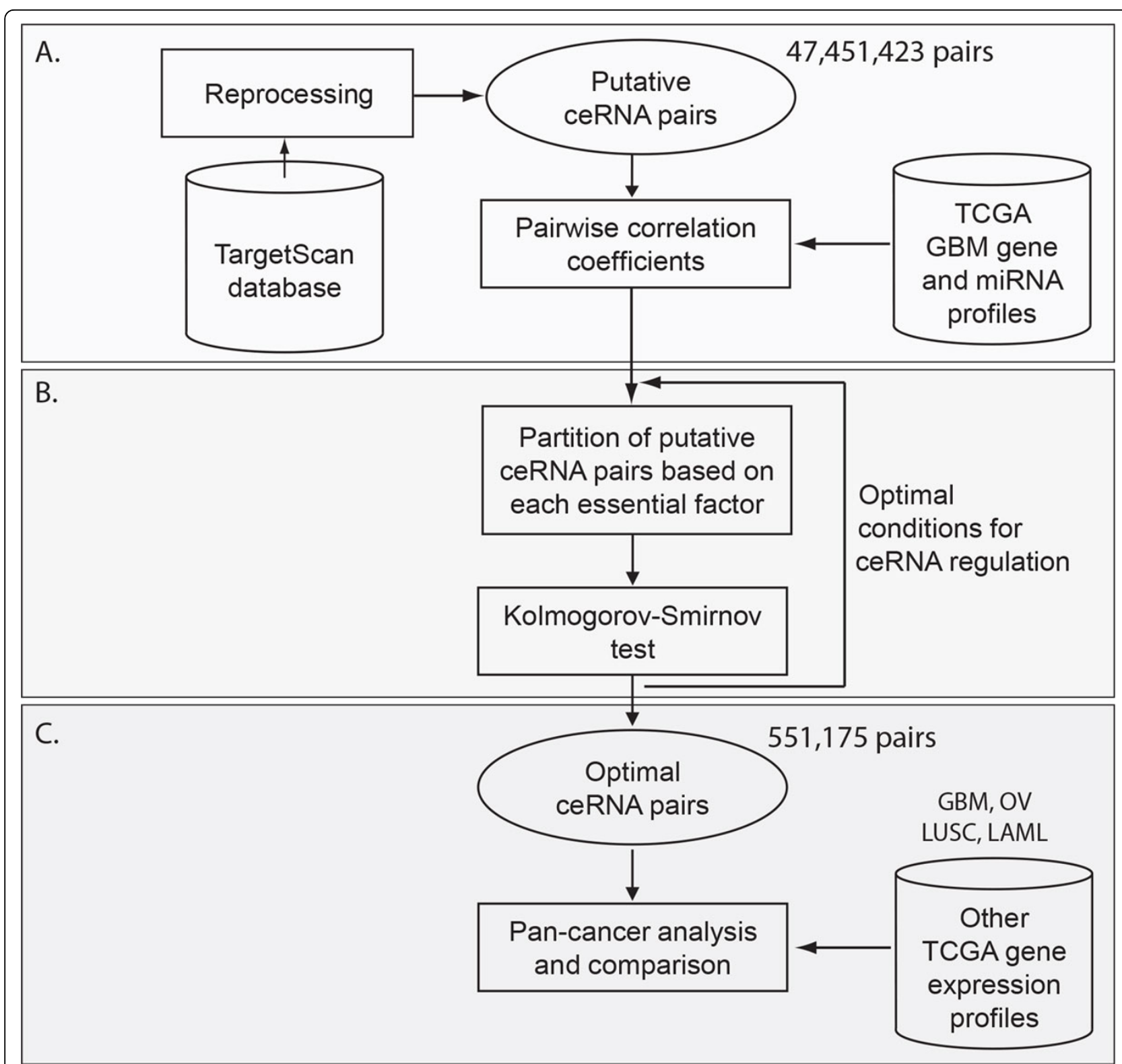

Figure 1 Analysis flowchart of this study. The present study is aimed to systematically explore optimal conditions and related biological functions of ceRNA regulation in GBM, and confer cancer type specific and independent effects. (A) First we defined 47,451,423 putative ceRNA pairs as pairs of genes (i.e., pairs of ceRNAs) sharing any number of predicted targeting miRNAs in the TargetScan database. Pairwise correlation coefficients of putative ceRNA pairs were computed in 481-sample TCGA GBM datasets. (B) Correlation coefficients were partitioned into groups based on the states of each essential factor of putative ceRNA pairs, followed by inter-group goodness-of-fit tests (K-S tests) that pinpointed the essential factors and optimal conditions for ceRNA regulation. (C) 551,175 pairs of ceRNAs fulfilling all of the identified optimal conditions were defined as optimal ceRNA pairs. In order to address differential and constitutive functions, we then included TCGA OV, LUSC, and LAML datasets and performed pan-cancer analysis.

conditions for intensified ceRNA regulation depend on the essential factors (Figure 1B). Here the intensified ceRNA regulation refers to the overall increased degree of coexpression of ceRNA pairs. Upon identification of optimal conditions with respect to the four essential factors, we defined the optimal ceRNA pairs from the putative ceRNAs, which satisfy all the four optimal conditions. We then performed functional annotation analysis to investigate biological processes and functions governed by optimal ceRNA regulation (Figure 1C). To further address the cancer type-specific and independent effects, we evaluated pairwise coexpression of the optimal ceRNA pairs identified from GBM in other TCGA cancer datasets, including 585-sample ovarian serous cystadenocarcinoma (OV) [19], 
133-patient lung squamous cell carcinoma (LUSC) [20], and 197-sample acute myeloid leukemia (LAML) [21]. The comprehensive results from the functional annotation level and from the enrichment of signaling pathways were reported in later sections below.

Increased size of miRNA program and number of miRNA program binding sites intensify ceRNA regulation in GBM The size of miRNA programs (number of common targeting miRNAs) among the $47,451,423$ putative ceRNA pairs ranged from 1 to 262, with quartiles of 2, 6, and 14 (Additional file 1: Figure $\mathrm{S} 1$ ). In order to dissect the association of size of miRPs with ceRNA regulation, we divided the putative ceRNA pairs into 4 groups based on size of miRPs, (i) 7,382,212 putative pairs $<25^{\text {th }}$-percentile, (ii) $15,289,167$ pairs within $25^{\text {th }}-50^{\text {th }}$ percentiles, (iii) $12,163,761$ pairs within $50^{\text {th }}-75^{\text {th }}$ percentiles, and (iv) $12,616,283$ pairs $\geq$ $75^{\text {th }}$-percentile, and compared the distributions of correlation coefficients across groups. Coexpression of ceRNA pairs was significantly elevated with increasing size of miRPs (K-S test $p$-value < computing precision of doubleprecision floating point, hereafter referred to as $p$-value $\sim 0$, between any two groups) while correlation coefficients of $13,043,077$ non-ceRNA pairs followed approximately the null random distribution (Figure 2A-B).

As defined in the Methods section, the number of miRNA program binding sites (\#miRPBS) was determined by summing up the total number of interacting sites of miRPs on the corresponding pairs of ceRNAs. Among all the putative ceRNA pairs the \#miRPBS fell in the range of 2-1,859 (histogram in Additional file 2: Figure S2). We grouped the putative ceRNA pairs based on the \#miRPBS with identical criteria as used in analyzing size of miRNA programs, resulting in 4 groups of $10,829,459,12,443,877$, $12,296,702$, and $11,881,385$ putative ceRNA pairs. With the K-S tests, significant $p$-values between any two groups ( $p$-value $\sim 0$ ) indicate that the number of miRP binding sites is positively associated with ceRNA coexpression (Figure 2C-D). Taken together, our data demonstrate that increased numbers of common targeting miRNAs as well as the abundance of binding sites intensify the strength of ceRNA regulation.

Strength of ceRNA regulation is dependent on expression levels of miRNA programs and ceRNAs in GBM

We reasoned that ceRNA regulation might depend on expression levels of miRNAs in miRPs. To test this hypothesis, we split the putative ceRNAs into 4 groups by the quartiles of expression levels of miRPs $(6.29,6.88$, and 7.52 in $\log 2$ scale; histogram in Additional file 3: Figure S3), resulting in $11,862,855$ ceRNA pairs in each group. Here the expression levels of miRPs were calculated by simply averaging the expression levels of miRNAs in miRPs for each ceRNA pair. All of the four groups (i.e., Q1 to Q4) showed significantly different distributions of correlation coefficients from non-ceRNA pairs and the Q3 group showed the most right-shifted distribution function (Figure 3A-B). Among the four groups, remarkably, the putative ceRNA pairs with median miRP expression levels (Q2 and Q3) exhibited higher correlation than ones belonging to Q1 and Q4 (K-S test $p$-value $\sim 0)$. This result was in agreement with the common assumption that both excessive abundance (loss of competition) and sparse availability (nothing to compete for) of miRNA transcripts will reduce regulation.

We further investigated whether ceRNA expression levels play a crucial role in governing ceRNA regulation. 3,653, 3,222 , and 3,343 genes with mean expression levels across the samples falling in the range of 5-35, 35-65, and 65-95 percentiles of all 10,872 genes analyzed, respectively, were annotated as low (L), medium $(\mathrm{M})$, and high $(\mathrm{H})$ expression states. We note that in the analysis we eliminated the genes within the bottom or top $5 \%$ expression levels, which may be largely attributed to background or saturation noise. Putative ceRNA pairs were then categorized based on expression states of their composed ceRNAs (see Table 1 for number of ceRNA pairs in each group). Interestingly, the results revealed high involvement of expression levels of ceRNAs in determining ceRNA regulation (Figure 3C). 4,551,383 (9.59\%) putative ceRNA pairs composed of two highly expressed genes showed significant coexpression $(\mathrm{H}-\mathrm{H})$ compared to other ceRNA pairs and non-ceRNA pairs (K-S statistics $=0.356$ and 0.378 and both $\mathrm{K}-\mathrm{S}$ test $p$-values $\sim 0$; Figure $3 \mathrm{D})$. Overall, the results suggest that ceRNA regulation is dependent on expression levels of both miRPs and ceRNAs. Remarkably, our observation from analysis of clinical microarray data agrees with Ala et al.'s data using a mathematical mass-action model in that optimal cellular conditions for ceRNA regulation depend on expression levels of miRNA programs as well as ceRNAs [13].

\section{Intertwined signaling among optimal ceRNAs is associated with essential cellular functions and disease pathways}

Observing that regulation strength is intensified in ceRNA pairs with a large size of miRP (Q4), large number of miRP binding sites (Q4), appropriate miRP expression levels (Q3), and high expression levels of both partner ceRNAs $(\mathrm{H}-\mathrm{H})$, we then defined 551,175 pairs of ceRNAs satisfying all of the four optimal conditions as optimal ceRNA pairs. The optimal ceRNA pairs accounted for only $1.16 \%$ of all putative ceRNA pairs, containing 2,405 optimal ceRNAs (22.12\%) of all 10,872 ceRNA genes. Additional file 4: Table S1 includes list of the optimal ceRNA pairs and summary of optimal ceRNAs. Pairwise coexpression of the optimal ceRNA pairs led other ceRNA pairs and non-ceRNAs by large margins $(\mathrm{K}-\mathrm{S}$ statistics $=0.391$ and 0.442 and both 


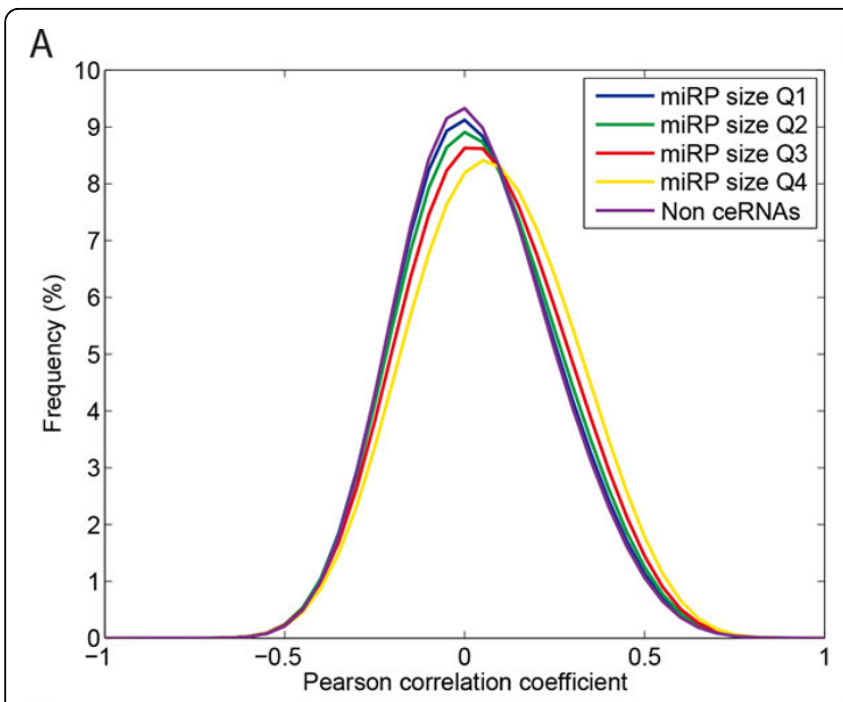

C

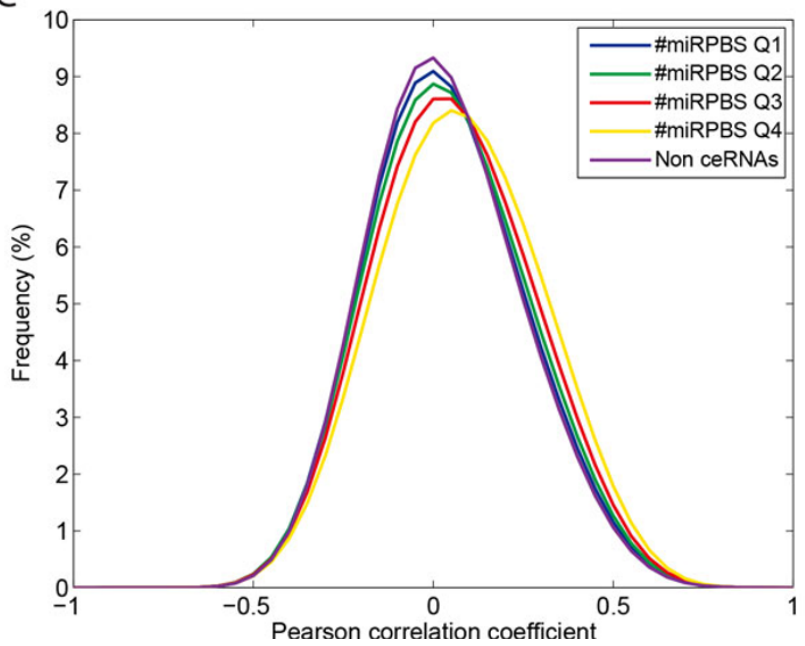

B

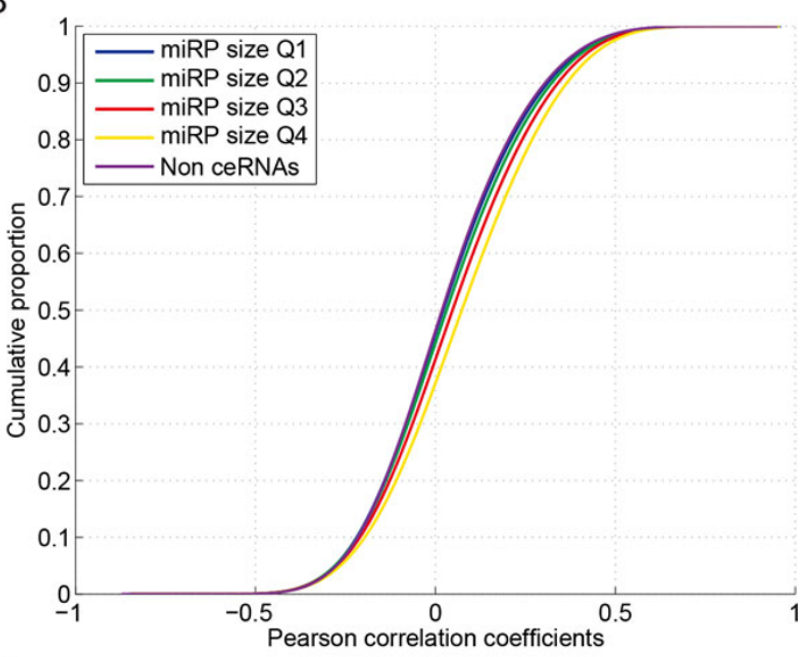

D

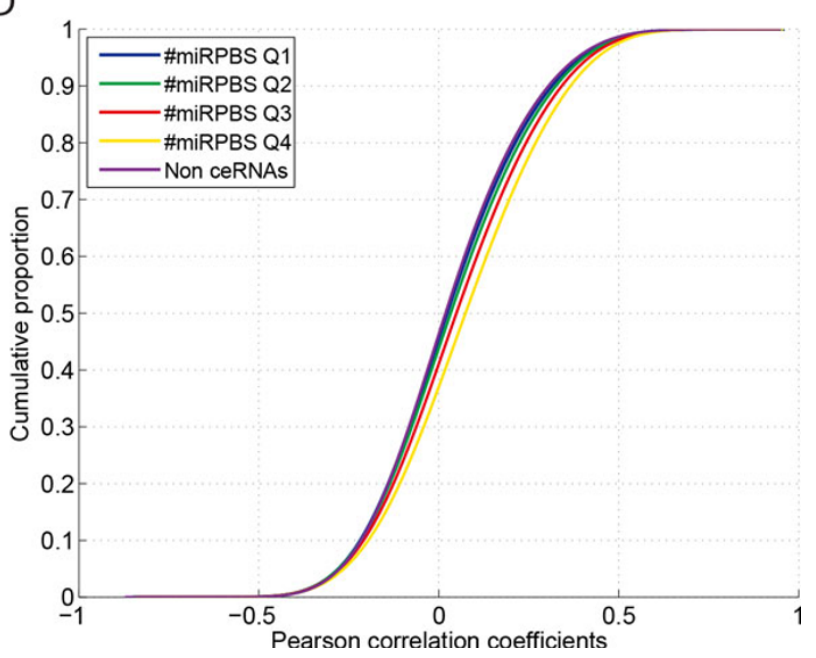

Figure 2 Effects of size of miRNA programs and number of miRNA program binding sites on ceRNA regulation. (A, B) Density functions and cumulative distribution functions of correlation coefficients of putative ceRNA pairs. The putative ceRNA pairs were divided into four groups by the quartiles of miRNA program sizes. (C, D) Density functions and cumulative distribution functions of correlation coefficients of putative ceRNA pairs, which were partitioned based on number of miRNA program binding sites.

K-S test $p$-values $\sim 0$, Figure $4 \mathrm{~A})$. In order to dissect higherorder properties of inter-ceRNA signaling, we merged the identified optimal ceRNA pairs and constructed the optimal ceRNA regulatory network (Figure 4B). On average each ceRNA directly interacted with up to $\sim 458$ optimal ceRNA partners, suggestive of the complex signaling maintained by ceRNA regulation. Connected to 1,480 first-order neighbors CDS2 (CDP-diacylglycerol synthase (phosphatidate cytidylyltransferase) 2) was found as the top hub ceRNA in the network, with the greatest number of first-order neighbors. Many of the top 20 hub genes were previously reported to be associated with cancer (8 genes), neurological diseases (9), hereditary disorders (9), or the function of cell death and survival (7) (data from database of Ingenuity Pathway Analysis (Qiagen Inc.), Table 2). Notably, the well-studied prognosis biomarker in cancers, SMAD family member 4 (SMAD4), regulated up to 1,382 optimal ceRNAs and was ranked $12^{\text {th }}$ in the hub ceRNA list. Functional annotation analysis indicated that the 2,405 optimal ceRNAs played crucial roles in the biological functions of intracellular transport (GO:0046907, Bonferroni adjusted $p$-value $=9.26 \times 10-18)$ and protein localization (GO:0008104, Bonferroni adjusted $p$-value $=7.88 \times$ 10-17). Figure 4C-D depicts complex intra-function ceRNA regulation within the two functions. For a more comprehensive overview of functional annotation, we utilized The Database for Annotation, Visualization and Integrated Discovery (DAVID) [22,23] to analyze the enriched clusters of Gene Ontology (GO) biological process and molecular function terms. The top five clusters of 


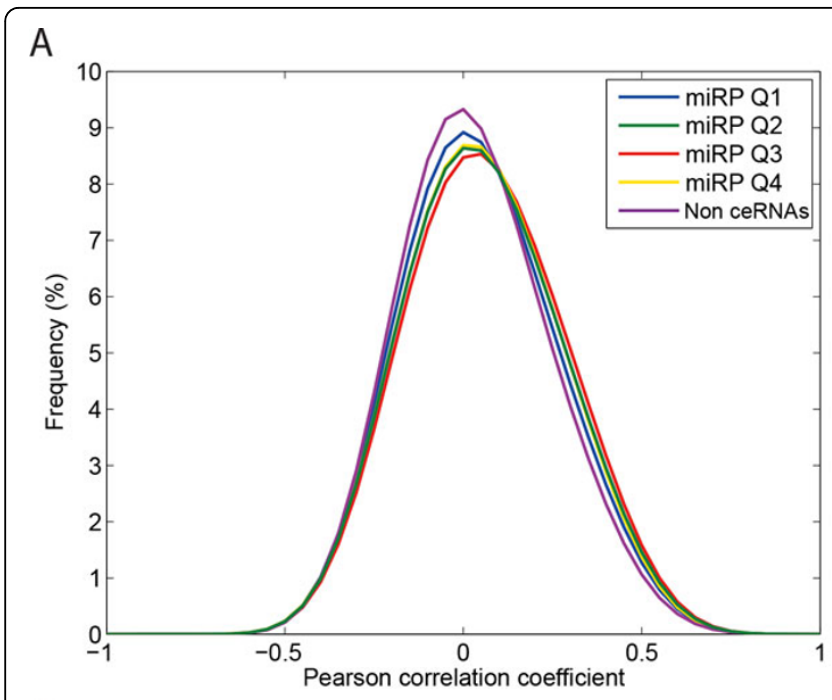

C

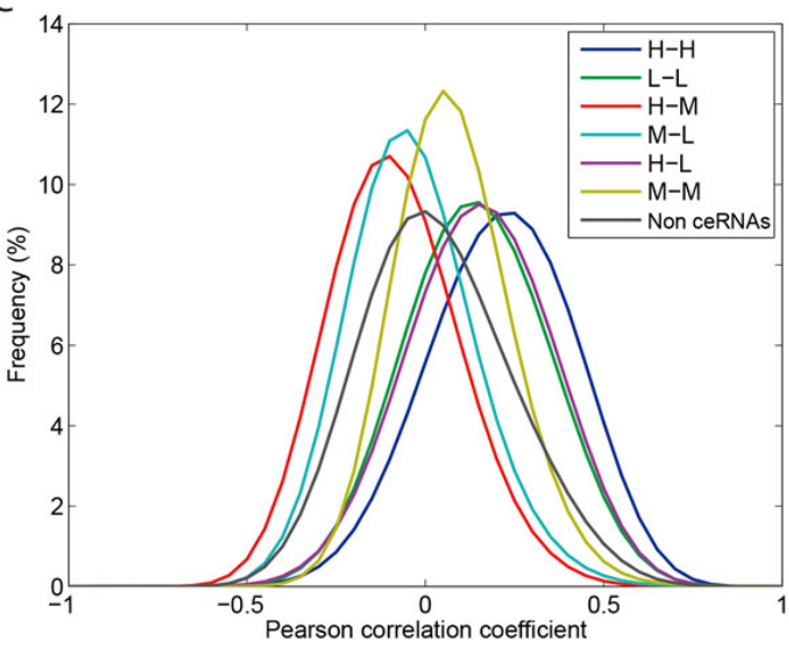

B

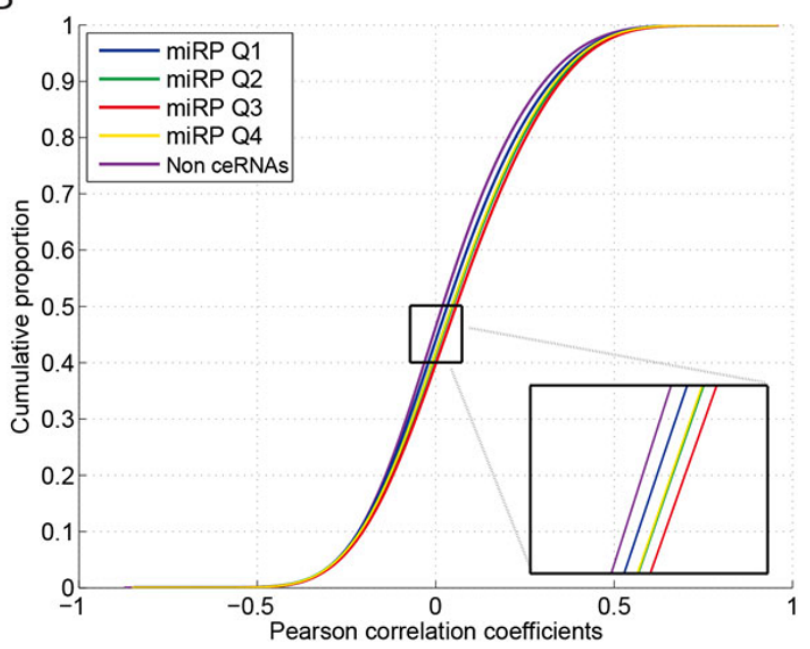

D

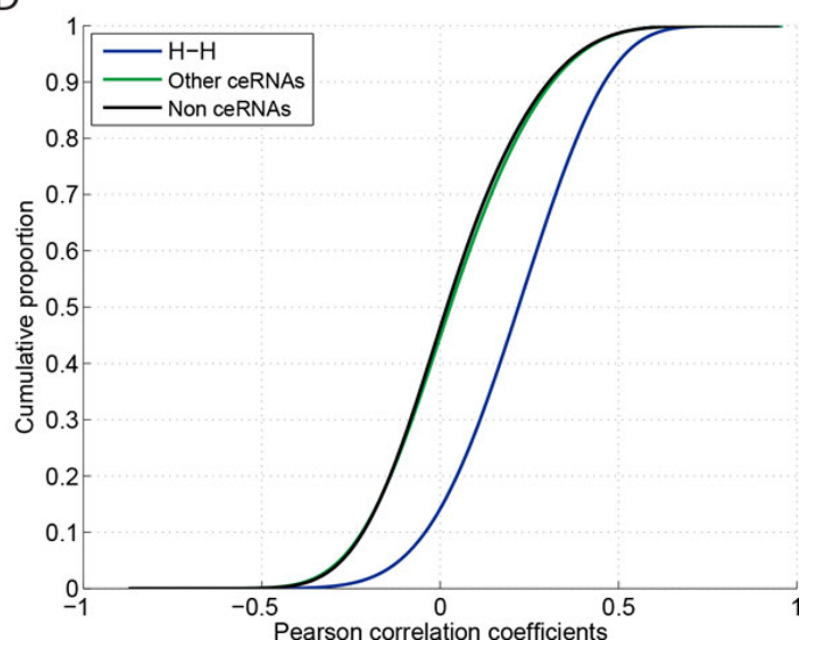

Figure 3 Effects of expression levels of miRNA programs and ceRNAs on ceRNA regulation. (A, B) Density functions and cumulative distribution functions of correlation coefficients of putative ceRNA pairs. The putative ceRNA pairs were split into groups by quartiles of miRNA programs expression levels. (C) Density functions of correlation coefficients of putative ceRNA pairs. Here the putative ceRNA pairs were categorized based on expression states (i.e., H, M, and L) of their composed ceRNAs. (D) Cumulative distribution functions of (C), focused on comparison of ceRNA pairs composed of two highly expressed genes $(\mathrm{H}-\mathrm{H})$ to other ceRNA pairs and non-ceRNA pairs.

functions were protein transport, protein catabolic process, vehicle-mediated transport, protein modification/ubiquitination, and regulation of translation (see Table 3 DAVID cluster scores $>5$ ). Taken together, our data indicate that optimal ceRNA regulation is highly involved in diseases and maintenance of essential cellular functions.
Pan-cancer analysis revealed dynamic ceRNA regulation among constitutive ceRNAs

For further conferring similarity/dissimilarity of ceRNA regulation and functions among different cancer types, we analyzed pairwise correlation coefficients of the 551,175 optimal ceRNA pairs in TCGA datasets of GBM,

Table 1 Number of ceRNA pairs in groups categorized based on expression states of their composed ceRNAs.

\begin{tabular}{cccc}
\hline & High-expression genes $(\mathbf{H})$ & Medium-expression genes (M) & Low-expression genes (L) \\
\hline High-expression genes (H) & $4551383(9.59 \%)$ & & \\
\hline Medium-expression genes (M) & $8262660(17.41 \%)$ & $3786914(7.98 \%)$ & $5654651(11.92 \%)$ \\
\hline Low-expression genes (L) & $10134010(21.36 \%)$ & $9302868(19.61 \%)$ & \\
\hline
\end{tabular}

The percentages were calculated with respect to the number of all putative ceRNA pairs $(47,451,423)$. 


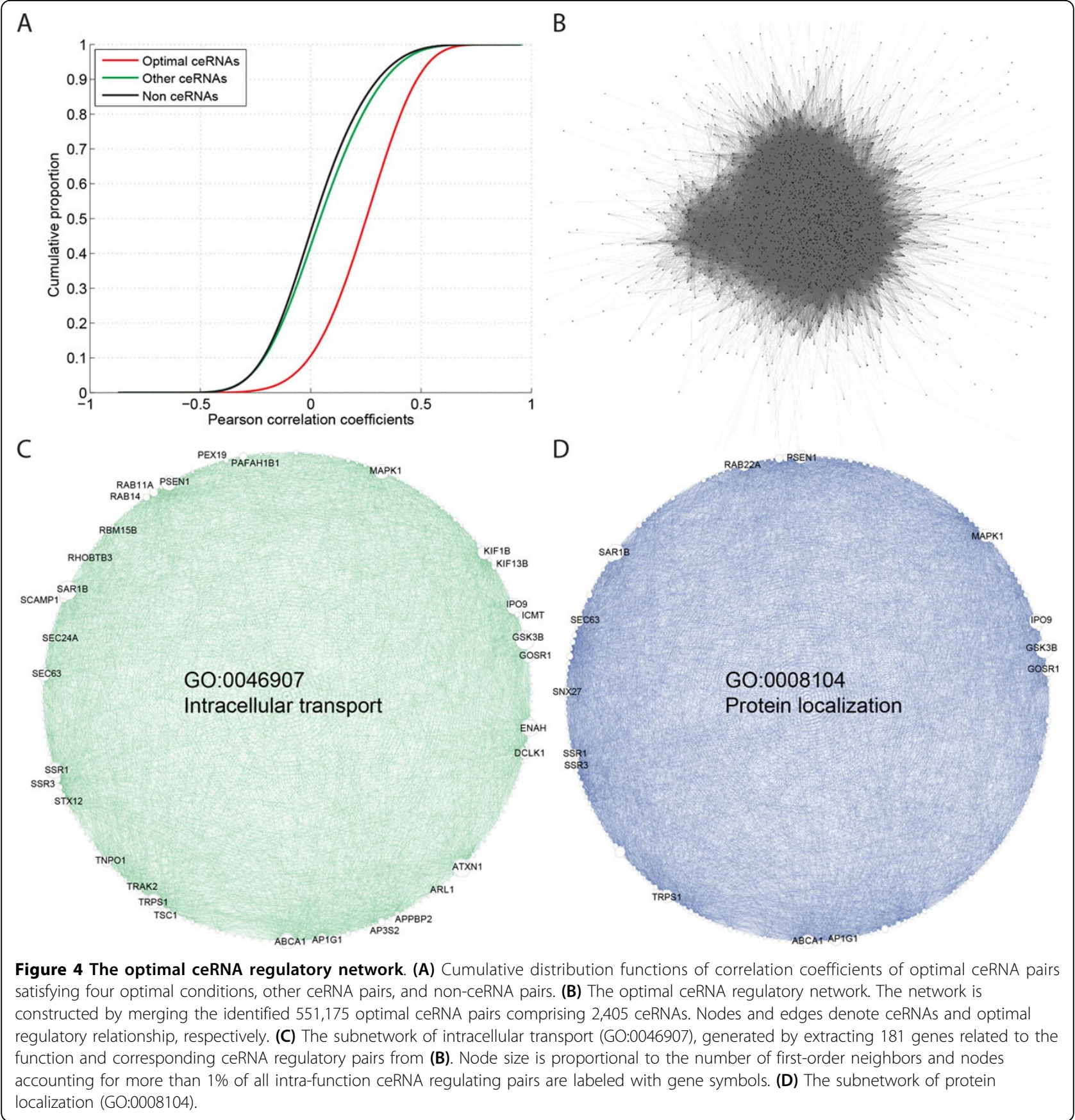

OV, LUSC, and LAML. Among the optimal ceRNA pairs, 452,718 (82.14\%), 358,359 (65.02\%), 239,886 (43.52\%), and $106,940(19.40 \%)$ pairs (hereafter referred to as core ceRNA pairs) exhibited significantly positive correlation coefficients (right-tail $p$-value $<0.05$ ) in GBM, OV, LUSC, and LAML, respectively. Notably, although numbers of significant regulating pairs changed immensely, the involved ceRNAs were largely similar among cancer types: 2,389 ( $99.33 \%$ out of 2,405 optimal ceRNAs), 2,377 (98.84\%), 2,353 (97.84\%), and 2,293 (95.34\%) ceRNAs (core ceRNAs) in four cancer types, with 2,278 in common (Figure 5A). For the most highly connected core ceRNAs in each cancer, LAML showed relatively distinctive results from the solid tumors, with 27 out of the 59 (45.76\%) LAML hub core ceRNAs appearing exclusively in LAML (Figure 5B). Detailed lists of core ceRNA pairs are tabulated in Additional file 5: Table S2. Also, the four sets of core ceRNAs shared highly identical enriched biological functions with the optimal ceRNAs (data not shown). Among the five clusters of GO terms associated 
Table 2 Top 20 hub genes of the optimal ceRNA network.

\begin{tabular}{|c|c|c|c|c|c|}
\hline $\begin{array}{l}\text { Hub } \\
\text { genes }\end{array}$ & $\begin{array}{l}\text { No. of first-order } \\
\text { neighbors }\end{array}$ & $\begin{array}{l}\text { Percentage of } \\
\text { total optimal } \\
\text { ceRNA pairs }\end{array}$ & Entrez gene name & Type $^{a}$ & Disease/Function $^{a}$ \\
\hline CDS2 & 1480 & $0.269 \%$ & $\begin{array}{c}\text { CDP-diacylglycerol synthase } \\
\text { (phosphatidate cytidylyltransferase) } \\
2\end{array}$ & enzyme & \\
\hline PARVA & 1470 & $0.267 \%$ & parvin, alpha & other & cancer; cell death and survival \\
\hline SLC1A2 & 1466 & $0.266 \%$ & $\begin{array}{c}\text { solute carrier family } 1 \text { (glial high } \\
\text { affinity glutamate transporter), } \\
\text { member } 2\end{array}$ & transporter & neurological disease; hereditary disorder \\
\hline NFIB & 1435 & $0.260 \%$ & nuclear factor $\mathrm{I} / \mathrm{B}$ & $\begin{array}{l}\text { transcription } \\
\text { regulator }\end{array}$ & \\
\hline SSR1 & 1421 & $0.258 \%$ & signal sequence receptor, alpha & other & \\
\hline GTF2H5 & 1407 & $0.255 \%$ & $\begin{array}{c}\text { general transcription factor } \mathrm{IH}, \\
\text { polypeptide } 5\end{array}$ & other & neurological disease; hereditary disorder \\
\hline SAR1B & 1398 & $0.254 \%$ & SAR1 homolog B (S. cerevisiae) & enzyme & hereditary disorder \\
\hline GSK3B & 1392 & $0.253 \%$ & glycogen synthase kinase 3 beta & kinase & $\begin{array}{l}\text { cancer; cell death and survival; neurological } \\
\text { disease; hereditary disorder }\end{array}$ \\
\hline HEG1 & 1390 & $0.252 \%$ & $\begin{array}{l}\text { heart development protein with } \\
\text { EGF-like domains } 1\end{array}$ & other & cancer \\
\hline ZNF148 & 1390 & $0.252 \%$ & zinc finger protein 148 & $\begin{array}{l}\text { transcription } \\
\text { regulator }\end{array}$ & cell death and survival \\
\hline EIF5 & 1387 & $0.252 \%$ & $\begin{array}{l}\text { eukaryotic translation initiation } \\
\text { factor } 5\end{array}$ & $\begin{array}{l}\text { translation } \\
\text { regulator }\end{array}$ & cancer \\
\hline SMAD4 & 1382 & $0.251 \%$ & SMAD family member 4 & $\begin{array}{l}\text { transcription } \\
\text { regulator }\end{array}$ & $\begin{array}{c}\text { cancer; cell death and survival; prognosis } \\
\text { biomarker; neurological disease; hereditary } \\
\text { disorder }\end{array}$ \\
\hline TCF4 & 1374 & $0.249 \%$ & transcription factor 4 & $\begin{array}{l}\text { transcription } \\
\text { regulator }\end{array}$ & $\begin{array}{c}\text { cell death and survival; neurological disease; } \\
\text { hereditary disorder }\end{array}$ \\
\hline QKI & 1366 & $0.248 \%$ & $\begin{array}{l}\text { QKI, KH domain containing, RNA } \\
\text { binding }\end{array}$ & other & $\begin{array}{l}\text { cancer; neurological disease; hereditary } \\
\text { disorder }\end{array}$ \\
\hline LSAMP & 1364 & $0.247 \%$ & $\begin{array}{l}\text { limbic system-associated } \\
\text { membrane protein }\end{array}$ & other & \\
\hline ATXN1 & 1354 & $0.246 \%$ & ataxin 1 & $\begin{array}{l}\text { transcription } \\
\text { regulator }\end{array}$ & $\begin{array}{l}\text { cancer; cell death and survival; neurological } \\
\text { disease; hereditary disorder }\end{array}$ \\
\hline DCP2 & 1354 & $0.246 \%$ & decapping mRNA 2 & enzyme & \\
\hline PSD3 & 1346 & $0.244 \%$ & $\begin{array}{l}\text { pleckstrin and Sec7 domain } \\
\text { containing } 3\end{array}$ & other & cancer; neurological disease \\
\hline SLC38A1 & 1340 & $0.243 \%$ & solute carrier family 38, member 1 & transporter & \\
\hline VAPB & 1340 & $0.243 \%$ & $\begin{array}{l}\text { VAMP (vesicle-associated } \\
\text { membrane protein)- associated } \\
\text { protein B and C }\end{array}$ & other & $\begin{array}{c}\text { cell death and survival; neurological disease; } \\
\text { hereditary disorder }\end{array}$ \\
\hline
\end{tabular}

with the optimal ceRNAs, the number of intra-function core ceRNA pairs varied massively (coefficients of variation all $>28.13 \%$, Table 3 ) while the number of comprising ceRNAs remained relatively stable (coefficients of variation ranging from $1.79 \%$ to $2.88 \%$, Table 3 ). The top (protein transport) and the $4^{\text {th }}$ (protein modification/ubiquitination) clusters were found with the largest changes in number of core ceRNA pairs and ceRNAs, respectively (see Additional file 6: Figure S4 and Additional file 7: Figure S5 for network visualization). Furthermore, our analyses revealed that, although massive re-wiring underlies ceRNA regulation among cancer types, the overall topology (recruitment of ceRNAs) of the core ceRNA networks was relatively stable, maintained mainly by a tiny subset of ceRNA regulating pairs with an extremely high degree of coexpression (Additional file 8: Figure S6). Incorporating these observations, we concluded that while the strength of ceRNA regulation is dynamic across cancer types, the essential biological functions governed by ceRNA regulation are stably retained. 
Table 3 Top 5 clusters of Gene Ontology terms enriched in the 2,405 optimal ceRNAs

\begin{tabular}{|c|c|c|c|c|c|c|c|c|}
\hline GO Term & $\begin{array}{l}\text { No. of } \\
\text { genes }\end{array}$ & $\begin{array}{c}\text { Bonferroni } \\
\text { adjusted } \\
P \text {-value } \\
\end{array}$ & $\begin{array}{l}\text { Total No. of optimal } \\
\text { ceRNA pairs/ceRNAs }\end{array}$ & $\begin{array}{l}\text { GBM } \\
\text { core }^{a}\end{array}$ & $\begin{array}{l}\text { OV } \\
\text { core }^{a}\end{array}$ & $\begin{array}{l}\text { LUSC } \\
\text { core }^{a}\end{array}$ & $\begin{array}{l}\text { LAML } \\
\text { core }^{a}\end{array}$ & $\begin{array}{l}\text { CV among } \\
\text { cancers }\end{array}$ \\
\hline \multicolumn{9}{|c|}{ Cluster 1 (Enrichment Score: 17.99) } \\
\hline GO:0046907 intracellular transport & 184 & $9.26 \mathrm{E}-18$ & & & & & & \\
\hline GO:0008104 protein localization & 225 & $7.88 \mathrm{E}-17$ & & & & & & \\
\hline GO:0015031 protein transport & 200 & 4.67E-16 & & & & & & \\
\hline $\begin{array}{l}\text { GO:0045184 establishment of protein } \\
\text { localization }\end{array}$ & 201 & $6.18 \mathrm{E}-16$ & $8229 / 261$ & $\begin{array}{c}7152 / \\
261\end{array}$ & $\begin{array}{l}6235 / \\
258\end{array}$ & $\begin{array}{c}3961 / \\
254\end{array}$ & $\begin{array}{l}2755 / \\
247\end{array}$ & $\begin{array}{l}34.85 \% / \\
2.06 \%\end{array}$ \\
\hline $\begin{array}{c}\text { GO:0070727 cellular macromolecule } \\
\text { localization }\end{array}$ & 126 & $2.75 \mathrm{E}-14$ & & & & & & \\
\hline GO:0034613 cellular protein localization & 124 & 1.09E-13 & & & & & & \\
\hline GO:0006886 intracellular protein transport & 111 & 3.17E-11 & & & & & & \\
\hline \multicolumn{9}{|c|}{ Cluster 2 (Enrichment Score: 8.27) } \\
\hline $\begin{array}{c}\text { GO:0009057 macromolecule catabolic } \\
\text { process }\end{array}$ & 180 & 2.95E-08 & & & & & & \\
\hline $\begin{array}{c}\text { GO:0044265 cellular macromolecule } \\
\text { catabolic process }\end{array}$ & 168 & $1.04 \mathrm{E}-07$ & & & & & & \\
\hline GO:0030163 protein catabolic process & 143 & $6.75 \mathrm{E}-06$ & & & & & & \\
\hline $\begin{array}{l}\text { GO:0043632 modification-dependent } \\
\text { macromolecule catabolic process }\end{array}$ & 134 & $8.41 \mathrm{E}-06$ & & & & & & \\
\hline $\begin{array}{c}\text { GO:0019941 modification-dependent } \\
\text { protein catabolic process }\end{array}$ & 134 & $8.41 \mathrm{E}-06$ & $4838 / 200$ & $\begin{array}{l}4418 / \\
200\end{array}$ & $\begin{array}{c}3836 / \\
197\end{array}$ & $\begin{array}{c}2379 / \\
193\end{array}$ & $\begin{array}{c}2049 / \\
191\end{array}$ & $\begin{array}{l}31.08 \% / \\
1.79 \%\end{array}$ \\
\hline $\begin{array}{c}\text { GO:0051603 proteolysis involved in cellular } \\
\text { protein catabolic process }\end{array}$ & 138 & $1.31 \mathrm{E}-05$ & & & & & & \\
\hline $\begin{array}{c}\text { GO:0044257 cellular protein catabolic } \\
\text { process }\end{array}$ & 138 & $1.85 \mathrm{E}-05$ & & & & & & \\
\hline $\begin{array}{c}\text { GO:0006511 ubiquitin-dependent protein } \\
\text { catabolic process }\end{array}$ & 68 & $8.96 \mathrm{E}-05$ & & & & & & \\
\hline GO:0006508 proteolysis & 177 & 1 & & & & & & \\
\hline \multicolumn{9}{|c|}{ Cluster 3 (Enrichment Score: 6.01) } \\
\hline GO:0016192 vesicle-mediated transport & 141 & $8.98 \mathrm{E}-08$ & & & & & & \\
\hline GO:0016044 membraneorganization & 93 & $3.70 \mathrm{E}-04$ & $3462 / 157$ & $\begin{array}{c}2882 / \\
155\end{array}$ & $\begin{array}{l}2519 / \\
156\end{array}$ & $\begin{array}{c}1607 / \\
149\end{array}$ & $\begin{array}{l}1208 / \\
147\end{array}$ & $\begin{array}{l}32.82 \% / \\
2.53 \%\end{array}$ \\
\hline GO:0010324 membrane invagination & 50 & 0.935246 & & & & & & \\
\hline GO:0006897 endocytosis & 50 & 0.935246 & & & & & & \\
\hline \multicolumn{9}{|c|}{ Cluster 4 (Enrichment Score: 5.67) } \\
\hline $\begin{array}{l}\text { GO:0032446 protein modification by small } \\
\text { protein conjugation }\end{array}$ & 45 & $6.85 E-05$ & & & & & & \\
\hline $\begin{array}{c}\text { GO:0070647 protein modification by small } \\
\text { protein conjugation or removal }\end{array}$ & 50 & $2.36 \mathrm{E}-04$ & & & & & & \\
\hline GO:0016567 protein ubiquitination & 40 & $7.30 \mathrm{E}-04$ & & & & & & \\
\hline $\begin{array}{c}\text { GO:0019787 small conjugating protein } \\
\text { ligase activity }\end{array}$ & 45 & 0.012039 & $607 / 67$ & $\begin{array}{l}555 / \\
67\end{array}$ & $\begin{array}{c}488 / \\
66\end{array}$ & $335 / 65$ & $266 / 62$ & $\begin{array}{l}28.13 \% / \\
2.88 \%\end{array}$ \\
\hline GO:0016881 acid-amino acid ligase activity & 51 & 0.020482 & & & & & & \\
\hline GO:0004842 ubiquitin-protein ligase activity & 39 & 0.084550 & & & & & & \\
\hline $\begin{array}{l}\text { GO:0016879 ligase activity, forming carbon- } \\
\text { nitrogen bonds }\end{array}$ & 53 & 0.213866 & & & & & & \\
\hline
\end{tabular}

\begin{tabular}{|c|c|c|c|c|c|c|c|c|}
\hline \multicolumn{9}{|c|}{ Cluster 5 (Enrichment Score: 5.10 ) } \\
\hline $\begin{array}{c}\text { GO:0010608 posttranscription al regulation } \\
\text { of gene expression }\end{array}$ & 59 & 0.001017 & & & & & & \\
\hline GO:0006417 regulation of translation & 40 & 0.036298 & $1312 / 105$ & $\begin{array}{c}1132 / \\
105\end{array}$ & $\begin{array}{r}961 / \\
101\end{array}$ & $643 / 99$ & $\begin{array}{r}480 / \\
100\end{array}$ & $\begin{array}{c}31.90 \% / \\
2.25 \%\end{array}$ \\
\hline $\begin{array}{c}\text { GO:0032268 regulation of cellular protein } \\
\text { metabolic process }\end{array}$ & 96 & 0.576893 & & & & & & \\
\hline
\end{tabular}

${ }^{a}$ Number of optimal ceRNA pairs/ceRNAs with significant positive correlation coefficients in corresponding cancer dataset, ${ }^{b}$ Coefficients of variation of number of core ceRNA pairs/ceRNAs among four cancer datasets. 

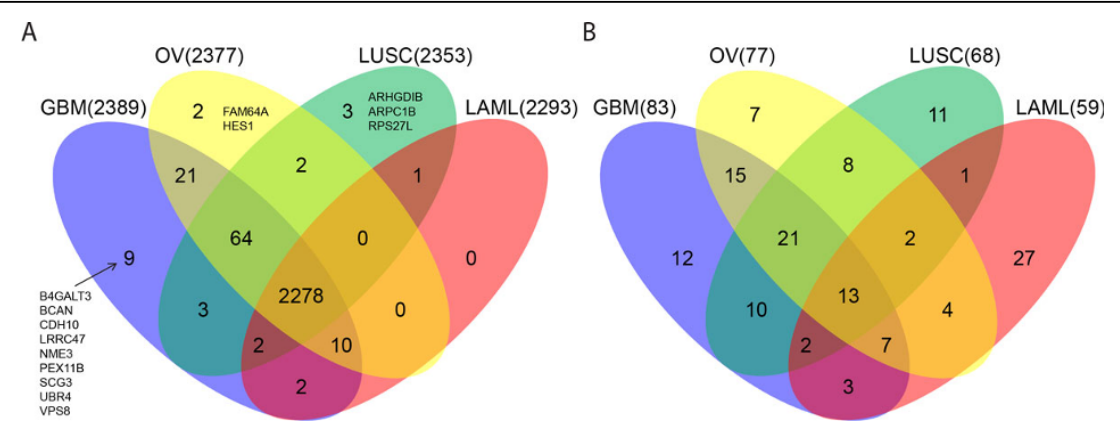

Figure 5 Venn diagrams of core ceRNAs among four cancer types. (A) Comparison of core ceRNAs in four cancer datasets. Core ceRNAs are genes that comprise the core ceRNA pairs with significant positive correlation coefficients in a cancer dataset. (B) Comparison of top hub core ceRNAs which collectively account for $10 \%$ of the total core ceRNA regulating pairs in corresponding cancer datasets.

\section{Discussion}

Besides the well-studied role of miRNAs in directly regulating gene expression, emerging evidence postulates that ceRNA regulation is an alternative mechanism through which miRNAs participate in gene regulation. Regulation of ceRNAs has been proved to govern essential biological functions in human development and diseases including cancer (reviewed in $[24,25]$ ). While recent studies have proved the dynamicity of ceRNA regulation among cellular conditions based on in silico and in vitro experiments $[13,14]$, comprehensive investigation into the strength of in vivo ceRNA regulation remains largely unexplored. Addressing this, in the present study we started by characterizing crucial factors in determination of optimal conditions using gene expression profiles derived from tumor specimens from TCGA. Our analyses indicated the dose effect of miRNA programs; i.e., increased size of miRNA programs as well as increased number of miRNA program binding sites enhance the competing relationship among genes and thus elevate inter-ceRNA coexpression. Furthermore, the expressional levels of both miRNA programs and ceRNAs affect ceRNA regulation and lead to statistically significant differences in distributions of correlation coefficients, suggestive of the existence of optimal molecular conditions in which ceRNA regulation prevails. Intermediate expression levels of miRNA programs allow efficient and effective competition, thus further optimize the power of ceRNA regulation; while varied expression levels of ceRNAs exhibited divergent effects on ceRNA regulation. Taken together, our analyses clearly demonstrated that ceRNA regulation highly depends on states of the essential factors and thus may involve complex and dynamic processes in vivo. Incorporating the optimal conditions of ceRNA regulation, we identified the optimal ceRNA pairs and revealed the biological functions significantly associated with protein transport, protein catabolic processes, and regulation of translation. These functions are all of essential significance in regular cellular routines, indicative of the indispensable involvement of ceRNA regulation in vivo. Recently, Denzler et al. assessed the ceRNA effect in hepatocytes and liver using quantitative biological experiments [26]. Our findings agree with their paper where it was concluded that ceRNA regulation is more likely to occur when both ceRNAs are highly expressed or miRNA binding sites are sufficient. Interestingly, analyzing the unusually highly expressed miRNA miR-122, they showed that coexpression of miR-122 target genes was achieved specifically at extremely high target site abundance. Our data further showed the dependence of ceRNA regulation on the essential factors and cancer types. Taken together, we elucidated that ceRNA regulation is a complex and sophisticated mechanism in vivo, thus difficult to be observed under some cellular conditions. Future biological studies may investigate it in detail and carry out essential clues to complex ceRNA regulation.

With the growing volume of DNA microarray and nextgeneration sequencing samples, pan-cancer analysis has unveiled both common and unique characteristics of genomic aberrations [15], expression profiles [27], oncogenic microRNAs [16], and secretome [28] across cancer types. This emerging research domain illuminates the tumor type-specific and independent molecular properties, and further contributes to enhanced understanding of tumorigenesis and progression. In this pioneer report, for better characterizing ceRNA in cancers, we extended the optimal ceRNA pairs identified from GBM to large datasets deposited in TCGA, including ovarian serous cystadenocarcinoma, lung squamous cell carcinoma, and acute myeloid leukemia datasets. Our results demonstrated that ceRNA regulatory networks are massively rewired across cancer types. Acute myeloid leukemia exhibited the most distinctive ceRNA pattern from other solid tumors. Remarkably, although the strength and wiring of ceRNA regulation changed immensely, the recruitment of genes into the ceRNA regulatory network is highly stable among cancer types; i.e., the highly intertwined ceRNA regulatory relationship enables genes to be effectively regulated by some of their ceRNA partners regardless of perturbations 
to cellular conditions. This property of ceRNA regulation stabilizes intra-function regulation and thus facilitates maintenance of essential biological functions in cells. With the increasing number of molecular profiles of cancers, future analysis may extend the analysis to more cancer types and provide universal landscape of ceRNA regulation.

In the present study, for each of four factors we attempted with the quartiles and specific percentiles to partition the putative ceRNAs into groups and compare inter-group distribution of correlation coefficients. For inferring more subtle changes in distribution of correlation coefficients, future work may use other methods that are capable of revealing local fluctuations of distribution functions. For defining putative ceRNA pairs, we employed prediction data from the miRNA-target gene prediction algorithm of TargetScan. TargetScan is a widely used prediction algorithm that takes into consideration both sequence complementarity (especially the seed regions of miRNAs) and conservativity of binding sites. There are still a handful of prediction algorithms, such as PicTar, based on genome-wide sequence alignment [29] and mirBridge utilizing gene set enrichment analysis [30]. While different prediction methods, as well as species-specific targeting, define dissimilar miRNA-target gene pairs, since our present report was aimed to investigate the systematic view of ceRNA regulation and its optimal conditions, out of simplicity we only employed the TargetScan algorithm. Indeed, ceRNAs with larger number of targeting miRNAs are expected to have more putative ceRNA partners, and thus account for a higher proportion of the 47 million putative ceRNA pairs. However, in the analysis of optimal conditions for each parameter, the calculation of correlation coefficients was based on "ceRNA pairs" instead of "ceRNAs". Furthermore, since TargetScan is one of the algorithms with the highest prediction precision (reviewed in [31]), in this study the putative ceRNA pairs were defined with high confidence, regardless of the numbers of their ceRNA partners. Thus, we reason that differences in the number of ceRNA partners among genes would not cause major systematic biases to our analysis.

Besides, here we adopted the biologically straightforward Pearson correlation as a measure of gene-gene coexpression, other methods such as mutual information and polynomial regression may provide alternatives for modeling non-linear properties of miRP-modulated coexpression of ceRNAs. In the report, out of simplicity we focused on "pairwise" relationship between ceRNA pairs. However, the competition for a set of miRNAs may not be exclusively limited to pairs of ceRNAs since a handful of ceRNAs can compete, fully or partially, for common targeting miRNAs. Realizing that one miRNA can target up to hundreds of mRNAs in the genome-wide scale, taking all these factors into account will exponentially complicate the problem and thus require more complex mathematical models.

\section{Conclusions}

Here we carried out a comprehensive investigation into optimal conditions for competing endogenous RNA regulation, associated biological functions, and pan-cancer effects of ceRNA regulation. Using TCGA GBM microarray datasets, we demonstrated that regulation between ceRNAs is dynamic, however the optimal conditions are quantifiable. The obtained optimal ceRNA regulatory network is associated with diseases pathways and essential cellular functions. Pan-cancer analysis revealed that while strength of ceRNA regulation is dynamic across cancer types, the highly intertwined ceRNA signaling stably maintains the essential functions it governs. Therefore, we expect the study presented here brings biological insights into the dynamicity and essential roles of ceRNA regulation.

\section{Methods}

\section{Microarray expression datasets}

Microarray datasets of glioblastoma multiforme (GBM) [17], ovarian serous cystadenocarcinoma (OV) [19], lung squamous cell carcinoma (LUSC) [20], and acute myeloid leukemia (LAML) [21] patients were downloaded from The Cancer Genome Atlas (TCGA) database. We extracted 481 GBM samples with paired miRNA and mRNA expression profiles from the datasets of 557sample Affymetrix Human Genome U133A Arrays and 505-sample Agilent $8 \times 15 \mathrm{~K}$ Human miRNA-specific microarrays. The OV, LUSC, and LAML datasets were composed of 585-sample Affymetrix Human Genome U133A Arrays, 133-sample Affymetrix Human Genome U133A Arrays, and 197-sample Affymetrix Human Genome U133 Plus 2.0 Arrays, respectively. We utilized TCGA Level 3 data, which were previously normalized and merged into gene- or miRNA-level expression values by TCGA, for consequent analysis in this study.

\section{MiRNA targeting genes}

For defining miRNA targeting genes we adopted miRNAtarget gene prediction data from the TargetScan database $[2,18]$, which predicts binding sites of miRNA families on targets based on the context scores. Genes with either conserved or poorly conserved binding sites for a miRNA were defined as targeting genes of interest for the corresponding miRNA. For each of the genes with multiple transcripts, only one transcript was selected as the representative transcript. Let $G$ and $M$ denote the total number of genes and miRNAs, respectively. The miRNAtarget gene information can be mathematically described in the $G$-by- $M$ miRNA-target matrix $\mathbf{T}$.

$$
\mathbf{T}=\left\{t_{g m}\right\}
$$

where $g=1, \ldots, G, m=1, \ldots, M$, and $t_{g m}$ is an indicator function, or $t_{g m}=1$ if $m^{\text {th }}$ miRNA binds to $g^{\text {th }}$ gene as 
defined above, otherwise, $t_{g m}=0$. Putative competitive endogenous RNA (ceRNA) pairs, defined as pairs of genes sharing common predicted binding miRNAs (i.e., miRNA programs or miRPs) according to previous reports $[9,11]$, correspond to non-zero elements in the putative ceRNA matrix $\mathrm{P}$, or

$$
P_{G \times G}=\mathbf{T} \times T^{\prime}
$$

where $\mathbf{T}^{\prime}$ is the transpose of $\mathbf{T}$. For each putative ceRNA pair, we calculated the number of miRNA program binding sites (\#miRPBS) by summing the numbers of binding sites of all miRNAs belonging to the miRP on the pair of ceRNAs.

\section{Statistical analysis}

The Pearson correlation coefficient was utilized to measure degree of coexpression between expression profiles of pair of ceRNAs. In order to identify the essential factors that affect ceRNA regulation, we compared distributions of correlation coefficients obtained from different sets of putative ceRNA pairs. Each set of ceRNA pairs was derived by varying the size of miRPs, number of miRNA binding sites, etc., all essential factors for determination of ceRNA pairs. The goodness-of-fit between two cumulative distribution functions (CDFs) was evaluated with the two-sample Kolmogorov-Smirnov test (K-S test). By measuring the K-S statistic as the maximum vertical distance of two CDFs, the K-S test statistically infers whether two sets of samples are drawn from the same distribution and thus provides a nonparametric measure for comparing two CDFs. The putative ceRNA pairs satisfying the optimal states of essential factors in the GBM dataset were selected and defined as the "optimal ceRNA pairs".

\section{Construction and visualization of ceRNA networks}

To confer higher-order signaling properties among ceRNA pairs, we first identified optimal ceRNA pairs and then constructed the ceRNA networks for various cancer types. Nodes and edges in the network denote ceRNAs and co-expressions between ceRNA pairs, respectively. We utilized the open source software Cytoscape [32] for visualization of the network.

\section{Additional material}

Additional file 1: Figure S1. Histogram of size of miRNA programs for all putative ceRNA pairs.

Additional file 2: Figure S2. Histogram of number of miRNA program binding sites for all putative ceRNA pairs.

Additional file 3: Figure S3. Histogram of expression levels of miRNA programs for all putative ceRNA pairs.

Additional file 4: Table S1. List of optimal ceRNA pairs and summary of optimal ceRNAs.
Additional file 5: Table S2. Lists of core ceRNA pairs and core ceRNAs in four cancer types.

Additional file 6: Figure S4. Subnetworks of core ceRNA pairs related to cluster 1 (protein transport, detailed list of GO terms in Table 3) in cancers.

Additional file 7: Figure S5. Subnetworks of core ceRNA pairs related to cluster 4 (protein modification/ubiquitination, detailed list of GO terms in Table 3) in cancers.

Additional file 8: Figure S6. Number of core ceRNAs versus number of core ceRNA pairs exhibiting significant positive correlation with the core ceRNA regulatory networks.

\section{Competing interests}

The authors declare that they have no competing interests.

\section{Authors' contributions}

All authors conceived the study together. YuC designed the analysis pipeline and carried out the data analysis. $\mathrm{TH}$ and $\mathrm{YiC}$ revised the study design. YuC drafted the manuscript. YuC, YiC, and EYC reviewed and edited the manuscript and all authors read and approved the final manuscript.

\section{Acknowledgements}

The study is supported partly by the Ministry of Science and Technology, Taiwan (grant IDs 103-2917-I-002-166 and 103-2320-B-002-065-MY3). The authors wish to thank Center of Genomic Medicine, National Taiwan University for financial support and computing servers. The authors also greatly appreciate the brilliant and constructive inputs into this study from researchers during the 2013 IEEE International Workshop on Genomic Signal Processing and Statistics.

\section{Declarations}

The publication costs for this article were funded by the Ministry of Science and Technology, Taiwan (MOST 103-2320-B-002-065-MY3).

This article has been published as part of BMC Genomics Volume 16 Supplement 4, 2015: Selected articles from the IEEE International Workshop on Genomic Signal Processing and Statistics (GENSIPS) 2013. The full contents of the supplement are available online at http://www. biomedcentral.com/bmcgenomics/supplements/16/S4.

\section{Authors' details}

${ }^{1}$ Graduate Institute of Biomedical Electronics and Bioinformatics, National Taiwan University, Taipei, Taiwan. ${ }^{2}$ Greehey Children's Cancer Research Institute, University of Texas Health Science Center at San Antonio, San Antonio, Texas, USA. ${ }^{3}$ Department of Medical Research, Taichung Veterans General Hospital, Taichung, Taiwan. ${ }^{4}$ Department of Epidemiology and Biostatistics, University of Texas Health Science Center at San Antonio, San Antonio, Texas, USA. ${ }^{5}$ Bioinformatics and Biostatistics Core, Center of Genomic Medicine, National Taiwan University, Taipei, Taiwan.

Published: 21 April 2015

\section{References}

1. Bartel DP, Chen CZ: Micromanagers of gene expression: the potentially widespread influence of metazoan microRNAs. Nat Rev Genet 2004, 5(5):396-400.

2. Lewis BP, Burge CB, Bartel DP: Conserved seed pairing, often flanked by adenosines, indicates that thousands of human genes are microRNA targets. Cell 2005, 120(1):15-20.

3. Croce CM: Causes and consequences of microRNA dysregulation in cancer. Nat Rev Genet 2009, 10(10):704-714.

4. Valastyan S, Reinhardt F, Benaich N, Calogrias D, Szasz AM, Wang ZC, Brock JE, Richardson AL, Weinberg RA: A pleiotropically acting microRNA, miR-31, inhibits breast cancer metastasis. Cell 2009, 137(6):1032-1046.

5. Oneyama C, Morii E, Okuzaki D, Takahashi Y, Ikeda J, Wakabayashi N, Akamatsu H, Tsujimoto M, Nishida T, Aozasa K, et al: MicroRNA-mediated upregulation of integrin-linked kinase promotes Src-induced tumor progression. Oncogene 2012, 31(13):1623-1635. 
6. Hu Z, Chen X, Zhao Y, Tian T, Jin G, Shu Y, Chen Y, Xu L, Zen K, Zhang C, et al: Serum microRNA signatures identified in a genome-wide serum microRNA expression profiling predict survival of non-small-cell lung cancer. Journal of clinical oncology : official journal of the American Society of Clinical Oncology 2010, 28(10):1721-1726.

7. Boeri M, Verri C, Conte D, Roz L, Modena P, Facchinetti F, Calabro E, Croce CM, Pastorino U, Sozzi G: MicroRNA signatures in tissues and plasma predict development and prognosis of computed tomography detected lung cancer. Proc Natl Acad Sci USA 2011, 108(9):3713-3718.

8. Kozomara A, Griffiths-Jones S: miRBase: integrating microRNA annotation and deep-sequencing data. Nucleic Acids Res 2011, 39(Database):D152-D157.

9. Karreth FA, Tay Y, Perna D, Ala U, Tan SM, Rust AG, DeNicola G, Webster KA Weiss D, Perez-Mancera PA, et al: In vivo identification of tumorsuppressive PTEN ceRNAs in an oncogenic BRAF-induced mouse model of melanoma. Cell 2011, 147(2):382-395.

10. Sumazin $P$, Yang $X$, Chiu HS, Chung WJ, lyer A, Llobet-Navas D, Rajbhandari $P$, Bansal M, Guarnieri P, Silva J, et al: An extensive microRNAmediated network of RNA-RNA interactions regulates established oncogenic pathways in glioblastoma. Cell 2011, 147(2):370-381.

11. Tay Y, Kats L, Salmena L, Weiss D, Tan SM, Ala U, Karreth F, Poliseno L, Provero P, Di Cunto F, et al: Coding-independent regulation of the tumor suppressor PTEN by competing endogenous mRNAs. Cell 2011, 147(2):344-357.

12. Chiu Y-C, Chuang EY, Hsiao T-H, Chen Y: Modeling competing endogenous RNA regulatory networks in glioblastoma multiforme. Bioinformatics and Biomedicine (BIBM), 2013 IEEE International Conference on: 18-21 Dec. 2013 2013, 201-204.

13. Ala U, Karreth FA, Bosia C, Pagnani A, Taulli R, Leopold V, Tay Y, Provero P, Zecchina R, Pandolfi PP: Integrated transcriptional and competitive endogenous RNA networks are cross-regulated in permissive molecular environments. Proc Natl Acad Sci USA 2013, 110(18):7154-7159.

14. Bosia C, Pagnani A, Zecchina R: Modelling Competing Endogenous RNA Networks. PLoS One 2013, 8(6):e66609.

15. Kandoth C, McLellan MD, Vandin F, Ye K, Niu B, Lu C, Xie M, Zhang Q, McMichael JF, Wyczalkowski MA, et al: Mutational landscape and significance across 12 major cancer types. Nature 2013, 502(7471):333-339.

16. Hamilton MP, Rajapakshe K, Hartig SM, Reva B, McLellan MD, Kandoth C, Ding L, Zack Tl, Gunaratne PH, Wheeler DA, et al: Identification of a pancancer oncogenic microRNA superfamily anchored by a central core seed motif. Nature communications 2013, 4:2730.

17. Cancer Genome Atlas Research N: Comprehensive genomic characterization defines human glioblastoma genes and core pathways. Nature 2008, 455(7216):1061-1068.

18. Garcia DM, Baek D, Shin C, Bell GW, Grimson A, Bartel DP: Weak seedpairing stability and high target-site abundance decrease the proficiency of Isy-6 and other microRNAs. Nature structural \& molecular biology 2011, 18(10):1139-1146.

19. Cancer Genome Atlas Research N: Integrated genomic analyses of ovarian carcinoma. Nature 2011, 474(7353):609-615.

20. Cancer Genome Atlas Research N: Comprehensive genomic characterization of squamous cell lung cancers. Nature 2012, 489(7417):519-525

21. Cancer Genome Atlas Research N: Genomic and epigenomic landscapes of adult de novo acute myeloid leukemia. N Engl J Med 2013, 368(22):2059-2074.

22. Dennis G, Sherman BT, Hosack DA, Yang J, Gao W, Lane HC, Lempicki RA: DAVID: Database for Annotation, Visualization, and Integrated Discovery. Genome biology 2003, 4(5):P3

23. Huang D, Sherman $B$, Lempicki R: Systematic and integrative analysis of large gene lists using DAVID bioinformatics resources. Nat Protoc 2009, 4:44-57.

24. de Giorgio A, Krell J, Harding V, Stebbing J, Castellano L: Emerging roles of competing endogenous RNAs in cancer: insights from the regulation of PTEN. Mol Cell Biol 2013, 33(20):3976-3982.

25. Tay $Y$, Rinn J, Pandolfi PP: The multilayered complexity of ceRNA crosstalk and competition. Nature 2014, 505(7483):344-352.

26. Denzler R, Agarwal V, Stefano J, Bartel DP, Stoffel M: Assessing the ceRNA hypothesis with quantitative measurements of miRNA and target abundance. Mol Cell 2014, 54(5):766-776.

27. Parikh N, Hilsenbeck S, Creighton CJ, Dayaram T, Shuck R, Shinbrot E, Xi L, Gibbs RA, Wheeler DA, Donehower LA: Effects of TP53 mutational status on gene expression patterns across 10 human cancer types. J Pathol 2014, 232(5):522-533.

28. Wu CC, Hsu CW, Chen CD, Yu CJ, Chang KP, Tai DI, Liu HP, Su WH, Chang YS, Yu JS: Candidate serological biomarkers for cancer identified from the secretomes of 23 cancer cell lines and the human protein atlas. Molecular \& cellular proteomics : MCP 2010, 9(6):1100-1117.

29. Krek A, Grun D, Poy MN, Wolf R, Rosenberg L, Epstein EJ, MacMenamin P, da Piedade I, Gunsalus KC, Stoffel M, et al: Combinatorial microRNA target predictions. Nat Genet 2005, 37(5):495-500.

30. Tsang JS, Ebert MS, van Oudenaarden A: Genome-wide dissection of microRNA functions and cotargeting networks using gene set signatures. Mol Cell 2010, 38(1):140-153.

31. Witkos TM, Koscianska E, Krzyzosiak WJ: Practical Aspects of microRNA Target Prediction. Current molecular medicine 2011, 11(2):93-109.

32. Smoot ME, Ono K, Ruscheinski J, Wang PL, Ideker T: Cytoscape 2.8: new features for data integration and network visualization. Bioinformatics 2011, 27(3):431-432.

doi:10.1186/1471-2164-16-S4-S1

Cite this article as: Chiu et al:: Parameter optimization for constructing competing endogenous RNA regulatory network in glioblastoma multiforme and other cancers. BMC Genomics 2015 16(Suppl 4):S1.

\section{Submit your next manuscript to BioMed Central and take full advantage of:}

- Convenient online submission

- Thorough peer review

- No space constraints or color figure charges

- Immediate publication on acceptance

- Inclusion in PubMed, CAS, Scopus and Google Scholar

- Research which is freely available for redistribution
C Biomed Central 